\title{
Continuity of projections of natural bundles
}

\author{
by WŁOdZimierz M. Mikulski (Kraków)
}

\begin{abstract}
This paper is a contribution to the axiomatic approach to geometric objects. A collection of a manifold $M$, a topological space $N$, a group homomorphism $E: \operatorname{Diff}(M) \rightarrow \operatorname{Homeo}(N)$ and a function $\pi: N \rightarrow M$ is called a quasi-natural bundle if (1) $\pi \circ E(f)=f \circ \pi$ for every $f \in \operatorname{Diff}(M)$ and (2) if $f, g \in \operatorname{Diff}(M)$ are two diffeomorphisms such that $f|U=g| U$ for some open subset $U$ of $M$, then $E(f)\left|\pi^{-1}(U)=E(g)\right| \pi^{-1}(U)$. We give conditions which ensure that $\pi: N \rightarrow M$ is continuous. In particular, if $(M, N, E, \pi)$ is a quasi-natural bundle with $N$ Hausdorff, then $\pi$ is continuous. Using this result, we classify (quasi) prolongation functors with compact fibres.
\end{abstract}

0. Introduction. Throughout this paper manifolds are assumed to be paracompact, finite-dimensional and without boundary.

The concept of a natural bundle was introduced by A. Nijenhuis [10] as a modern approach to the classical theory of geometrical objects (see [1]). A natural bundle (over $n$-dimensional manifolds) is a covariant functor $F$ from the category of $n$-dimensional $C^{\infty}$ manifolds and $C^{\infty}$ embeddings into the category of $C^{\infty}$ locally trivial fibre bundles and $C^{\infty}$ bundle mappings such that:

(1) for every $n$-dimensional $C^{\infty}$ manifold $M, F M$ is a locally trivial fibre bundle over $M$;

(2) for every $C^{\infty}$ embedding $\varphi: M \rightarrow N$ of $n$-dimensional manifolds, $F \varphi: F M \rightarrow F N$ covers $\varphi$ and for any $x \in M, F \varphi$ maps diffeomorphically the fibre $F_{x} M$ onto the fibre $F_{\varphi(x)} N$;

(3) $F$ is regular in the following sense: If $\varphi: U \times M \rightarrow N$ is a $C^{\infty}$ mapping (where $U$ is an open subset of $\mathbb{R}^{k}$ ) such that for every $t \in U$, $\varphi_{t}: M \rightarrow N, \varphi_{t}(x)=\varphi(t, x)$, is an embedding, then the mapping

$$
U \times F M \ni(t, y) \rightarrow F \varphi_{t}(y) \in F N
$$

is of class $C^{\infty}$.

1991 Mathematics Subject Classification: Primary 55R65.

Key words and phrases: natural bundle, quasi-natural bundle, regular quasi-natural bundle, locally determined associated space, quasi-prolongation functor. 
The functor $T$ associating with each $n$-manifold $M$ the tangent bundle $T M$ and with each embedding $\varphi: M \rightarrow N$ the differential $d \varphi: T M \rightarrow T N$ is an example of a natural bundle. An equivalent formulation of an interesting result of Palais and Terng [11] is that every natural bundle is isomorphic to a fibre bundle associated with an $r$-frame bundle and whose standard fibre is an $L_{n}^{r}$ space (cf. [4, p. 13]).

The definition of a locally determined associated space was introduced by Epstein and Thurston [2] as a generalization of natural bundles. Let $n \geq 1$ be a fixed natural number and let $q \geq 1$ be a fixed integer or infinity. A locally determined associated space is a functor $E$ associating with each $n$-dimensional $C^{q}$ manifold $M$ a topological space $E M \neq \emptyset$ and a continuous mapping $\pi_{M}: E M \rightarrow M$ and with each $C^{q}$ embedding $\varphi: M \rightarrow N$ of $n$-manifolds a homeomorphism between $E M$ and $\pi_{N}^{-1}(\operatorname{im}(\varphi))$ such that $\pi_{N} \circ$ $E(\varphi)=\varphi \circ \pi_{M}$.

In [2], the authors proved that if $E$ is a locally determined associated space such that $E \mathbb{R}^{n}$ is a locally compact second countable Hausdorff space, then $E$ is isomorphic to a topological fibre bundle associated with an $r$-frame bundle and whose standard fibre is an $L_{n}^{r}$ space. (In [8], we extended the result of Epstein and Thurston to the situation when $E \mathbb{R}^{n}$ is not locally compact.) The result of Epstein and Thurston implies that the regularity condition in the definition of a natural bundle is a consequence of the other conditions in the definition.

The purpose of this paper is to give an answer to the following question: Is the condition " $\pi_{M}$ is continuous" in the definition of locally determined associated spaces a consequence of the other conditions of the definition (see Problem in [2, p. 236])? If $E M$ is a Hausdorff space, the answer is affirmative. In fact, we obtain a more general result.

Let us begin with the definition of a quasi-natural bundle.

DEFINITION 0.1. Let $r \geq 1$ be an integer or infinity or $r=\omega$ and let $n \geq 1$ be a natural number. A collection $(M, N, E, \pi, r, n)$ of $r, n$ and of

- an $n$-dimensional $C^{r}$ manifold $M$,

- a topological space $N \neq \emptyset$,

- a group homomorphism $E: \operatorname{Diff}(M) \rightarrow \operatorname{Homeo}(N)$ of the group of all global $C^{r}$ diffeomorphisms of $M$ onto $M$ into the group of all homeomorphisms of $N$ onto $N$,

- a function $\pi: N \rightarrow M$,

is called a quasi-natural bundle if the following two conditions are satisfied:

(1) for every $\varphi \in \operatorname{Diff}(M), \pi \circ E(\varphi)=\varphi \circ \pi$,

(2) if $f, g \in \operatorname{Diff}(M)$ are such that $f|U=g| U$ for some open subset $U$ of $M$, then $E(f)\left|\pi^{-1}(U)=E(g)\right| \pi^{-1}(U)$. 
We have the following obvious example of quasi-natural bundles.

EXAMPLE 0.1. Let $E$ be a locally determined associated space over $n$-dimensional $C^{r}$ manifolds and let $M$ be an $n$-dimensional $C^{r}$ manifold. It is clear that $\left(M, E M, E \mid \operatorname{Diff}(M), \pi_{M}, r, n\right)$ is a quasi-natural bundle.

In Section 1, conditions are given under which $\pi: N \rightarrow M$ is continuous, where $(M, N, E, \pi, r, n)$ is a quasi-natural bundle. In particular, we deduce the following two theorems.

TheOREM 0.1. Let $(M, N, E, \pi, r, n)$ be a quasi-natural bundle. Suppose that $N$ is a Hausdorff space and $r \geq 1$ is an integer or infinity. Then $\pi$ is continuous.

TheOREM 0.2. Let $(M, N, E, \pi, r, n)$ be a quasi-natural bundle. If $r \geq 1$ is an integer or infinity, then the set $\{v \in N: \pi$ is continuous at $v\}$ is open in $N$.

In Example 2.1, we present a quasi-natural bundle $(\mathbb{R}, N, E, \pi, \omega, 1)$ such that: (i) $N$ is a metric space and (ii) $\pi$ is not continuous. Therefore, Theorem 0.1 is not true in the analytic situation.

If $N$ is a $C^{r}$ manifold, we have the following natural question: Is $\pi$ : $N \rightarrow M$ of class $C^{r}$ ? It is a difficult question and I have only been able to give a partial answer. We introduce the following definition.

DeFinition 0.2. Let $(M, N, E, \pi, \infty, n)$ be a quasi-natural bundle. We say it is regular if the following three conditions are satisfied:

(1) $N$ is a manifold of class $C^{\infty}$;

(2) if $\varphi \in \operatorname{Diff}(M)$, then $E(\varphi): N \rightarrow N$ is of class $C^{\infty}$;

(3) if $\varphi: U \times M \rightarrow M$ is a $C^{\infty}$ mapping (where $U$ is an open subset of $\mathbb{R}^{k}$ ) such that for every $t$ in $U, \varphi_{t}: M \rightarrow M, \varphi_{t}(x)=\varphi(t, x)$, is a diffeomorphism, then the mapping

$$
U \times N \ni(t, y) \rightarrow E\left(\varphi_{t}\right)(y) \in N
$$

is of class $C^{\infty}$.

In Section 3, we prove the following theorem.

TheOREM 0.3. Let $(M, N, E, \pi, \infty, n)$ be a regular quasi-enatural bundle. Assume that $M$ and $N$ are connected. Then the set $\{v \in N$ : there exists a neighbourhood $V$ of $v$ such that $\pi \mid V$ is of class $\left.C^{\infty}\right\}$ is dense in $N$.

Similarly to the definition of prolongation functors in the sense of [6] we introduce the following definitions of quasi-prolongation functors.

DEFINITION 0.3. Let $r \geq 1$ be an integer or infinity or $r=\omega$. A quasiprolongation functor over (positive-dimensional) $C^{r}$ manifolds is a covariant functor $F$ associating with each positive-dimensional $C^{r}$ manifold $M$ a 
topological space $F M \neq \emptyset$ and a function $\pi_{M}: F M \rightarrow M$ and with each $C^{r}$ mapping $f: M \rightarrow N$ a continuous mapping $F f: F M \rightarrow F N$ such that:

(1) for every $C^{r}$ mapping $f: M \rightarrow N, \pi_{N} \circ F f=f \circ \pi_{M}$;

(2) if $\varphi: M \rightarrow N$ is a $C^{r}$ diffeomorphism onto an open subset of $N$, then $F \varphi: F M \rightarrow \pi_{N}^{-1}(\operatorname{im}(\varphi))$ is a homeomorphism.

Definition 0.4. Let $F$ be a quasi-prolongation functor over $C^{\infty}$ manifolds. We say that $F$ is regular if the following conditions are satisfied:

(1) for every $C^{\infty}$ manifold $M, F M$ is a $C^{\infty}$ manifold;

(2) for every $C^{\infty}$ mapping $f: M \rightarrow N$ the mapping $F f: F M \rightarrow F N$ is of class $C^{\infty}$

(3) if $\varphi: M \rightarrow N$ is a $C^{\infty}$ diffeomorphism onto an open subset, then $F \varphi: F M \rightarrow \pi_{N}^{-1}(\operatorname{im}(\varphi))$ is also a diffeomorphism. (By Theorem 0.1, $\pi_{N}$ is continuous, and so $\pi_{N}^{-1}(\operatorname{im}(\varphi))$ is open in $F N$.)

In Section 4, we prove the following theorem.

THEOREM 0.4. (I) Let $F$ be a quasi-prolongation functor over $C^{r}$ manifolds. Suppose that:

(1) $r$ is a natural number or infinity;

(2) for each $n, F \mathbb{R}^{n}$ has a countable basis;

(3) for each $C^{r}$ manifold $M, F M$ is Hausdorff;

(4) for each $n$ the fibre $\pi_{\mathbb{R}^{n}}^{-1}(0)$ is compact.

Then there exists a compact set $G$ such that $F$ is isomorphic to the trivial quasi-prolongation functor $(\mathrm{I}) \times G$ which associates with each $C^{r}$ manifold $M$ the space $M \times G$ and the mapping $p_{M}: M \times G \rightarrow M, p_{M}(x, y)=x$, and with each $C^{r}$ mapping $f$ the mapping $f \times \mathrm{id}_{G}$.

(II) Let $F$ be a regular quasi-prolongation functor. Assume that:

(1) for each $n, F \mathbb{R}^{n}$ has a countable basis;

(2) for each $n, \pi_{\mathbb{R}^{n}}^{-1}(0)$ is compact.

Then there exists a $C^{\infty}$ compact manifold $G$ such that $F$ is $C^{\infty}$ isomorphic to the trivial regular quasi-prolongation functor ( ) $\times G$ over $C^{\infty}$ manifolds.

Remark. We say that quasi-prolongation functors $F^{1}, F^{2}$ (resp. regular quasi-prolongation functors $F^{1}, F^{2}$ ) over $C^{r}$ (resp. $C^{\infty}$ ) manifolds are isomorphic $\left(C^{\infty}\right.$ isomorphic) if for every $C^{r}$ (resp. $\left.C^{\infty}\right)$ manifold $M$ there exists a homeomorphism (resp. a $C^{\infty}$ diffeomorphism) $I_{M}: F^{1} M \rightarrow F^{2} M$ such that:

(1) for every $C^{r}$ (resp. $\left.C^{\infty}\right)$ manifold $M, \pi_{M}^{2} \circ I_{M}=\pi_{M}^{1}$, where $\pi_{M}^{i}$ : $F^{i} M \rightarrow M(i=1,2)$ are the projections; 
(2) for each $C^{r}$ (resp. $C^{\infty}$ ) mapping $f: M \rightarrow N$ of $C^{r}$ (resp. $C^{\infty}$ ) manifolds, $I_{N} \circ F^{1} f=F^{2} f \circ I_{M}$.

1. Continuity of projections of quasi-natural bundles. In this section we will prove Theorems 0.1 and 0.2 .

Let $X$ be a topological space and let $Y \subset X$. We say that $Y$ is Hausdorff in $X$ iff any two distinct points in $Y$ have disjoint open neighbourhoods in $X$. It is clear that if $h: X \rightarrow X$ is a homeomorphism and $Y$ is Hausdorff in $X$, then $h(Y)$ is also Hausdorff in $X$.

Let $(M, N, E, \pi, r, n)$ be a quasi-natural bundle. Throughout this section we use the following notations:

$$
\operatorname{Orb}(v)=\{E(f)(v): f \in \operatorname{Diff}(M)\}, \quad v \in N ;
$$

for every $x \in M$,

$$
\begin{array}{r}
D^{-}(x)=\{f \in \operatorname{Diff}(M): f(x)=x \text { and there exists a chart }(U, g, x) \\
\text { such that } \left.g(x)=0 \text { and } \operatorname{det}\left(d_{0}\left(g \circ f \circ g^{-1}\right)\right)<0\right\} ;
\end{array}
$$

finally, for every $y \in M$, denote by $\operatorname{Comp}(M, y)$ the connected component of $M$ which contains $y$.

Theorems 0.1 and 0.2 are simple consequences of the following proposition.

Proposition 1.1. Let $(M, N, E, \pi, r, n)$ be a quasi-natural bundle and let $v \in N$. Suppose that $r \geq 1$ is an integer or infinity. Then the following conditions are equivalent:

(1) $\pi$ is continuous at $v$;

(2) if $w \in \operatorname{Orb}(v)$, then $\pi$ is continuous at $w$;

(3) for all $u, w \in \operatorname{Orb}(v)$, the condition $\pi(u) \neq \pi(w)$ implies that $\{u, w\}$ is Hausdorff in $N$;

(4) there exists $w \in \operatorname{Orb}(v)-\pi^{-1}(\pi(v))$ such that:

(a) $\pi(w) \in \operatorname{Comp}(M, \pi(v))$,

(b) $\{v, w\}$ is Hausdorff in $N$, and

(c) if $D^{-}(\pi(v)) \neq \emptyset$, then there exists $\varphi \in D^{-}(\pi(v))$ such that $\{E(\varphi)(v), w\}$ is Hausdorff in $N$;

(5) there exists an open (in $N$ ) neighbourhood $V$ of $v$ such that $\pi \mid V$ is continuous.

In the proof of Proposition 1.1 we shall use the following lemmas:

LEMma 1.1. Let $r \geq 1$ be an integer or infinity. Let $M$ be an $n$ dimensional manifold of class $C^{r}$ and let $x \in M$. Let $(U, g, x)$ be a chart of $M$ at $x$ such that $g(U)=\mathbb{R}^{n}$ and $g(x)=0$. If $y \in \operatorname{Comp}(M, x)$, then there 
exists a $C^{r}$ diffeomorphism $f: M \rightarrow M$ such that $\operatorname{germ}_{x}(f)=\operatorname{germ}_{x}\left(\operatorname{id}_{M}\right)$ and $f(y) \in U$.

Lemma 1.2. Let $(M, N, E, \pi, r, n)$ be a quasi-natural bundle such that $r \geq 1$ is an integer or infinity. Let $u, u^{\prime}, w \in N$ be such that:

(1) $u^{\prime} \in \operatorname{Orb}(u) \cap \pi^{-1}(\pi(u))$,

(2) $w \in \operatorname{Orb}(u)-\pi^{-1}(\pi(u))$

(3) $\{u, w\}$ is Hausdorff in $N$,

(4) if $D^{-}(\pi(u)) \neq \emptyset$, then there is a $\psi \in D^{-}(\pi(u))$ such that $\{E \psi(u), w\}$ is Hausdorff in $N$.

Then $\left\{u^{\prime}, w\right\}$ is also Hausdorff in $N$.

Proof of Lemma 1.1. Let

$A=\{z \in \operatorname{Comp}(M, x):$ there exists $f \in \operatorname{Diff}(M)$ such that $f(z) \in U$ and $\left.\operatorname{germ}_{x}(f)=\operatorname{germ}_{x}\left(\operatorname{id}_{M}\right)\right\}$.

We have to prove that $A=\operatorname{Comp}(M, x)$. It is clear that $U \subset A$. Thus it is sufficient to show that $A-\{x\}$ and $\operatorname{Comp}(M, x)-A$ are open in $\operatorname{Comp}(M, x)$.

For each $z \in A$, let $f_{z} \in \operatorname{Diff}(M)$ be such that $f_{z}(z) \in U$ and $\operatorname{germ}_{x}\left(f_{z}\right)=$ $\operatorname{germ}_{x}\left(\operatorname{id}_{M}\right)$. For each $t \in M-\{x\}$, let $\left(U_{t}, g_{t}\right)$ be a chart of $M$ at $t$ such that $g_{t}\left(U_{t}\right)=\mathbb{R}^{n}, g_{t}(t)=0$ and $x \in M-U_{t}$. For each $z \in U_{t}$ and $t \in M-\{x\}$, let $h_{t, z} \in \operatorname{Diff}(M)$ be such that $h_{t, z}(t)=z$ and $\operatorname{germ}_{x}\left(h_{t, z}\right)=\operatorname{germ}_{x}\left(\operatorname{id}_{M}\right)$ (see [5]).

Let $z \in A-\{x\}$. We shall show that $U_{z} \subset A-\{x\}$. Let $t \in U_{z}$. Then $f_{z} \circ\left(h_{z, t}\right)^{-1} \in \operatorname{Diff}(M), f_{z} \circ\left(h_{z, t}\right)^{-1}(t)=f_{z}(z) \in U$ and $\operatorname{germ}_{x}\left(f_{z} \circ\right.$ $\left.\left(h_{z, t}\right)^{-1}\right)=\operatorname{germ}_{x}\left(\operatorname{id}_{M}\right)$, and thus $t \in A-\{x\}$.

Let now $z \in \operatorname{Comp}(M, x)-A$. We shall show that $U_{z} \cap A=\emptyset$. Assume the contrary. Let $t \in U_{z} \cap A$. Then $f_{t} \circ h_{z, t} \in \operatorname{Diff}(M), f_{t} \circ h_{z, t}(z)=f_{t}(t) \in U$ and $\operatorname{germ}_{x}\left(f_{t} \circ h_{z, t}\right)=\operatorname{germ}_{x}\left(\operatorname{id}_{M}\right)$, and hence $z \in A$.

Proof of Lemma 1.2. Fix $f \in \operatorname{Diff}(M)$ such that $f(\pi(u))=\pi(u)$ and $u^{\prime}=E(f)(u)$, and a chart $(U, g)$ of $M$ at $\pi(u)$ such that $g(U)=\mathbb{R}^{n}$, $g(\pi(u))=0$ and $\pi(w) \in M-U$. Consider two cases.

(I) $\operatorname{det}\left(d_{0}\left(g \circ f \circ g^{-1}\right)\right)>0$. Then there exists $F \in \operatorname{Diff}(M)$ such that $\operatorname{germ}_{\pi(u)}(F)=\operatorname{germ}_{\pi(u)}(f)$ and $\operatorname{germ}_{\pi(w)}(F)=\operatorname{germ}_{\pi(w)}\left(\operatorname{id}_{M}\right)($ see $[5])$. Definition 0.1 ensures that $E(F)(w)=w$ and $E(F)(u)=E(f)(u)=u^{\prime}$; since $\{u, w\}$ is Hausdorff in $N$ and $E(F)$ is a homeomorphism, $\left\{u^{\prime}, w\right\}$ is also Hausdorff in $N$.

(II) $\operatorname{det}\left(d_{0}\left(g \circ f \circ g^{-1}\right)\right)<0$. Then $f \in D^{-}(\pi(u))$, i.e. $D^{-}(\pi(u)) \neq \emptyset$. By the assumptions $\{E(\psi)(u), w\}$ is Hausdorff in $N$ for some $\psi \in D^{-}(\pi(u))$. Replacing $u$ by $E(\psi)(u)$ and $f$ by $f \circ \psi^{-1}$ we apply Case (I) to conclude the proof. 
Proof of Proposition 1.1. (1) $\Rightarrow(2)$ is a simple consequence of Definition 0.1. $(2) \Rightarrow(3)$ and $(5) \Rightarrow(1)$ are obvious.

$(3) \Rightarrow(4)$. Let $(U, g)$ be a chart of $M$ at $\pi(v)$ such that $g(U)=\mathbb{R}^{n}$ and $g(\pi(v))=0$. Let $y \in U-\{\pi(v)\}$. There exist $h \in \operatorname{Diff}(M)$ and a compact set $K \subset U$ such that $h(\pi(v))=y$ and $h\left|M-K=\operatorname{id}_{M}\right| M-K$. Let $w:=E(h)(v)$. It is clear that $w \in \operatorname{Orb}(v)-\pi^{-1}(\pi(v))$ and $\pi(w)=$ $h(\pi(v)) \in U \subset \operatorname{Comp}(M, \pi(v))$. Thus $\{v, w\}$ is Hausdorff in $N$. Similarly, if $\varphi \in D^{-}(\pi(v))$, then $\pi(E(\varphi)(v))=\pi(v) \neq \pi(w)$, and so $\{E(\varphi)(v), w\}$ is Hausdorff in $N$.

The proof of $(4) \Rightarrow(1)$ is more complicated. Assume the contrary. Then $\pi$ is not continuous at $w$. Let $(U, g)$ be a chart of $M$ at $\pi(w)$ such that $g(U)=\mathbb{R}^{n}, g(\pi(w))=0$ and $\pi(W) \not \subset U$ for every $W \in \operatorname{top}(N, w)$, where $\operatorname{top}(N, w)$ is the set of all open neighbourhoods of $w$. By Lemma 1.1, there is an $f \in \operatorname{Diff}(M)$ such that $f(\pi(v)) \in U$ and $\operatorname{germ}_{\pi(w)}(f)=\operatorname{germ}_{\pi(w)}\left(\operatorname{id}_{M}\right)$. Moreover, there are $h \in \operatorname{Diff}(M)$ and a compact subset $K$ of $U$ such that $h\left|M-K=\operatorname{id}_{M}\right| M-K$ and $h(\pi(w))=f(\pi(v))$ (see [5]).

First assume that $E(f)(v), E(h)(w)$ and $w$ satisfy the assumptions of Lemma 1.2 (with $E(f)(v)$ and $E(h)(w)$ playing the roles of $u$ and $u^{\prime}$, respectively). Then $\{E(h)(w), w\}$ is Hausdorff in $N$, and hence there exist $W_{1} \in \operatorname{top}(N, E(h)(w))$ and $W_{2} \in \operatorname{top}(N, w)$ such that $W_{1} \cap W_{2}=\emptyset$. Since $E(h): N \rightarrow N$ is a homeomorphism, we can find $W \in \operatorname{top}(N, w)$ such that $W \subset W_{2}$ and $E(h)(W) \subset W_{1}$. Recalling that $\pi(\widetilde{W}) \not \subset U$ for every $\widetilde{W} \in \operatorname{top}(N, w)$, we deduce that $W-\pi^{-1}(U) \neq \emptyset$. On the other hand, since $\pi^{-1}(M-K) \supset N-\pi^{-1}(U)$ and $E(h)\left|\pi^{-1}(M-K)=\operatorname{id}_{N}\right| \pi^{-1}(M-K)$, we get $E(h)\left|N-\pi^{-1}(U)=\operatorname{id}_{N}\right| N-\pi^{-1}(U)$. Hence $\emptyset=W_{1} \cap W_{2} \supset$ $E(h)\left(W-\pi^{-1}(U)\right) \cap\left(W-\pi^{-1}(U)\right)=W-\pi^{-1}(U) \neq \emptyset$, a contradiction.

It remains to prove that $E(f)(v), E(h)(w)$ and $w$ satisfy the assumptions of Lemma 1.2. It is clear that $\operatorname{Orb}(E(h)(w))=\operatorname{Orb}(w)=\operatorname{Orb}(v)=$ $\operatorname{Orb}(E(f)(v))$ as $w \in \operatorname{Orb}(v)$. On the other hand, since $f$ is a bijection, $h(\pi(w))=f(\pi(v)), \pi(w) \neq \pi(v)$ and $\operatorname{germ}_{\pi(w)}(f)=\operatorname{germ}_{\pi(w)}\left(\operatorname{id}_{M}\right)$, we have $\pi(E(h)(w))=h(\pi(w))=f(\pi(v))=\pi(E(f)(v)), \pi(w)=f(\pi(w)) \neq$ $f(\pi(v))=\pi(E(f)(v))$ and $\{E(f)(v), w\}=E(f)(\{v, w\})$. Hence $E(h)(w) \in$ $\operatorname{Orb}(E(f)(v)) \cap \pi^{-1}(\pi(E(f)(v))), w \in \operatorname{Orb}(E(f)(v))-\pi^{-1}(\pi(E(f)(v)))$ and (since $\{v, w\}$ is Hausdorff in $N)\{E(f)(v), w\}$ is Hausdorff in $N$.

If $q \in D^{-}(f(\pi(v)))$, then $f^{-1} \circ q \circ f \in D^{-}(\pi(v))$ (i.e. $\left.D^{-}(\pi(v)) \neq \emptyset\right)$, and therefore (by the assumption on $w$ ) there exists $\varphi \in D^{-}(\pi(v))$ such that $\{E(\varphi)(v), w\}$ is Hausdorff in $N$. It is obvious that $f \circ \varphi \circ f^{-1} \in$ $D^{-}(f(\pi(v)))$ and $\left\{E\left(f \circ \varphi \circ f^{-1}\right)(E(f)(v)), w\right\}=E(f)(\{E(\varphi)(v), w\})$, and thus $\left\{E\left(f \circ \varphi \circ f^{-1}\right)(E(f)(v)), w\right\}$ is Hausdorff in $N$.

$(1) \Rightarrow(5)$. We can assume $(2)-(4)$ to hold. Fix $w \in\left(\operatorname{Orb}(v)-\pi^{-1}(\pi(v))\right)$ $\cap \pi^{-1}(\operatorname{Comp}(M, \pi(v)))$. If $D^{-}(\pi(v)) \neq \emptyset$, we fix $\psi \in D^{-}(\pi(v))$. Let $f \in$ 
$\operatorname{Diff}(M)$ be such that $w=E(f)(v)$. Let $W \in \operatorname{top}(M, \pi(v))$ be such that $\pi(w) \in M-\operatorname{cl}(W)$ and let $(U, g)$ be a chart of $M$ at $\pi(v)$ such that $g(U)=$ $\mathbb{R}^{n}, g(\pi(v))=0, U \subset W$ and $\psi(U) \subset W$. Since $\pi$ is continuous at $v$ and $w$, there are $V_{1} \in \operatorname{top}(N, v)$ and $V_{2} \in \operatorname{top}(N, w)$ such that $\pi\left(V_{1}\right) \subset U$ and $\pi\left(V_{2}\right) \subset M-\operatorname{cl}(W)$. Let $V \in \operatorname{top}(N, v)$ be such that $V \subset V_{1}$ and $E(f)(V) \subset V_{2}$.

We shall prove that $\pi \mid V$ is continuous. Let $\widetilde{v} \in V$ and $\widetilde{w}:=E(f)(\widetilde{v})$. We shall prove that $\widetilde{w}$ satisfies (4) (with $\widetilde{v}$ and $\widetilde{w}$ playing the roles of $v$ and $w$, respectively).

We see that $\pi(\widetilde{v}) \in \pi(V) \subset \pi\left(V_{1}\right) \subset U \subset W$ and $\pi(\widetilde{w}) \in \pi(E(f)(V)) \subset$ $\pi\left(V_{2}\right) \subset M-\operatorname{cl}(W)$. Therefore $\widetilde{w} \in \operatorname{Orb}(\widetilde{v})-\pi^{-1}(\pi(\widetilde{v}))$.

It is clear that $\pi(\widetilde{v}) \in U \subset \operatorname{Comp}(M, \pi(v))$. Then $\pi(\widetilde{w})=f(\pi(\widetilde{v})) \in$ $f(\operatorname{Comp}(M, \pi(v)))=\operatorname{Comp}(M, f(\pi(v)))=\operatorname{Comp}(M, \pi(w))$, and thus (as $\pi(w) \in \operatorname{Comp}(M, \pi(v))) \pi(\widetilde{w}) \in \operatorname{Comp}(M, \pi(\widetilde{v}))$.

Since $\widetilde{v} \in V \subset V_{1}$ and $\widetilde{w} \in E(f)(V) \subset V_{2}$ and $\pi\left(V_{1}\right) \cap \pi\left(V_{2}\right)=\emptyset,\{\widetilde{v}, \widetilde{w}\}$ is Hausdorff in $N$.

Let $h \in \operatorname{Diff}(M)$ be such that $h\left|M-U=\operatorname{id}_{M}\right| M-U$ and $h(\pi(v))=$ $\pi(\widetilde{v})$. Assume $\eta \in D^{-}(\pi(\widetilde{v}))$. Then $h^{-1} \circ q \circ h \in D^{-}(\pi(v))$, and then $D^{-}(\pi(v)) \neq \emptyset$. Let $\varphi:=h \circ \psi \circ h^{-1}$. Of course, $\varphi \in D^{-}(\pi(\widetilde{v}))$. We shall prove that $\{E(\varphi)(\widetilde{v}), \widetilde{w}\}$ is Hausdorff in $N$. It is sufficient to prove that $E(\varphi)(V) \cap E(f)(V)=\emptyset$. We know that $E(h) \mid \pi^{-1}(M-\operatorname{cl}(W))=$ $\operatorname{id}_{N} \mid \pi^{-1}(M-\operatorname{cl}(W))$, as $M-\operatorname{cl}(W) \subset M-W \subset M-U$. On the other hand, $E\left(h^{-1}\right)(V) \subset \pi^{-1}(U)$, for if $x \in V$, then $\pi(x) \in \pi(V) \subset \pi\left(V_{1}\right) \subset U$, and thus $\pi\left(E\left(h^{-1}\right)(x)\right)=h^{-1}(\pi(x)) \in U$. Therefore $($ as $\psi(U) \subset W)$ we have

$$
\begin{aligned}
E(f)(V) \cap E(\varphi)(V) & \subset V_{2} \cap E(\varphi)(V) \\
& \subset \pi^{-1}(M-\operatorname{cl}(W)) \cap E(h) \circ E(\psi) \circ E\left(h^{-1}\right)(V) \\
& \subset E(h)\left(\pi^{-1}(M-\operatorname{cl}(W))\right) \cap E(h) \circ E(\psi)\left(\pi^{-1}(U)\right) \\
& =E(h)\left(\pi^{-1}(M-\operatorname{cl}(W)) \cap E(\psi)\left(\pi^{-1}(U)\right)\right) \\
& \subset E(h)\left(\pi^{-1}(M-\operatorname{cl}(W)) \cap \pi^{-1}(W)\right)=\emptyset .
\end{aligned}
$$

We have the following interesting application of Theorem 0.1. Let $M$ be an $n$-dimensional $C^{r}$ manifold, where $n \geq 1$ and $r \geq 1$ is an integer or infinity. Let $\operatorname{top}(M)$ be the topology on $M$. We say that a topology $\tau$ on $M$ is natural iff any $\varphi \in \operatorname{Diff}(M)$ is a homeomorphism with respect to $\tau$. Of course, $\operatorname{top}(M)$ is natural.

Corollary 1.1. Let $M$ be as above and let $\tau$ be a Hausdorff natural topology on $M$. Then $\operatorname{top}(M) \subset \tau$.

Proof. Putting $N=(M, \tau), E: \operatorname{Diff}(M) \rightarrow \operatorname{Homeo}(N), E(\varphi)=\varphi$, and $\pi: N \rightarrow M, \pi=\mathrm{id}_{M}$, we obtain a quasi-natural bundle. Therefore $\pi$ is continuous, and thus top $(M) \subset \tau$. 
Rem ark. Let $M, N, \pi, r, n, \operatorname{Diff}(M), \operatorname{Homeo}(N)$ be as in Definition 0.1. Let $D$ be a subgroup in $\operatorname{Diff}(M)$ and let $E: D \rightarrow \operatorname{Homeo}(N)$ be a group homomorphism. The collection $(M, N, E, \pi, r, n, D)$ is called a D-quasi-natural bundle if the conditions of Definition 0.1 are satisfied with $D$ playing the role of Diff $(M)$. We see that Proposition 1.1 (and hence Theorems 0.1 and 0.2 ) with $D$ playing the role of $\operatorname{Diff}(M)$ is true for $D$-quasi-natural bundles provided $D$ satisfies the following conditions:

A) for any chart $(U, g)$ of $M$ at $x$ such that $g(U)=\mathbb{R}^{n}, g(x)=0$, and $y \in U$ there exist a compact set $K \subset U$ and $h \in D$ such that $h \mid M-K=$ $\operatorname{id}_{M} \mid M-K$ and $h(x)=y$,

B) for any chart $(U, g)$ of $M$ at $x$ and $f \in D-D^{-}(x)$ with $f(x)=x$ there exist a compact set $K \subset U$ and $h \in D$ such that $h=f$ near $x$ and $h\left|M-K=\operatorname{id}_{M}\right| M-K$.

For example, if either $D \subset \operatorname{Diff}(M)$ is the subgroup of all diffeomorphisms equal to the identity map outside a compact subset, or $M$ is oriented and $D$ is the subgroup of all orientation preserving diffeomorphisms, then $D$ satisfies the above conditions A) and B).

2. Counterexamples. In this section we present some counterexamples.

In connection with Theorem 0.1 we present the following example of a quasi-natural bundle $(M, N, E, \pi, \omega, 1)$, where $M$ is a connected analytic manifold, $N$ is a second countable metrizable space, $E: \operatorname{Diff}(M) \rightarrow$ $\operatorname{Homeo}(N)$ is a group homomorphism of the group of all analytic diffeomorphisms of $M$ onto $M$ into the group of all homeomorphisms of $N$ onto $N$ and $\pi: N \rightarrow M$ is not continuous.

EXAMPLE 2.1. Let $M=\mathbb{R}$ and let $N$ be the set of all global analytic mappings of $\mathbb{R}$ into $\mathbb{R}$. There is an injection $I: N \rightarrow \mathbb{R}^{\mathbb{N}}$ given by $I(f)=$ $\left(f(1), f\left(\frac{1}{2}\right), f\left(\frac{1}{3}\right), \ldots\right)$. We equip $N$ with the topology induced by $I$, i.e. $U \subset N$ is open iff $U=I^{-1}(V)$ for some $V$ open in $\mathbb{R}^{\mathbb{N}}$, where $\mathbb{R}^{\mathbb{N}}$ has the Tikhonov topology. Define $E: \operatorname{Diff}(M) \rightarrow \operatorname{Homeo}(N)$ by $E(g)(f)=g \circ f$. Let $\pi: N \rightarrow M$ be given by $\pi(f)=f(0)$. It is clear that $(M, N, E, \pi, \omega, 1)$ is a quasi-natural bundle. Since $f_{n}:=\left(n^{-n}+\left(\operatorname{id}_{\mathbb{R}}\right)^{2}\right)^{-n} \rightarrow 1$ (in $N$ ) and $\pi\left(f_{n}\right)=1 / n \nrightarrow \pi(1)=1$, we see that $\pi$ is not continuous at 1 .

In connection with Theorem 0.2 we give the following example.

ExAmple 2.2. Let $M=\mathbb{R}$. We equip $N:=\mathbb{R} \times \mathbb{R}$ with a topology defined as follows: $U \subset N$ is open if and only if either $U=N$ or $U=V \times\{0\}$ for some $V$ open in $\mathbb{R}$. Let $r=\infty$. Define $E: \operatorname{Diff}(M) \rightarrow \operatorname{Homeo}(N)$ by $E(f)(x, y)=(f(x), y)$. Let $\pi: N \rightarrow M$ be the projection onto the first 
factor. It is obvious that $(M, N, E, \pi, \infty, 1)$ is a quasi-natural bundle, but the set $\{v \in N: \pi$ is continuous at $v\}$ is not closed in $N$.

EXAmple 2.3. Let $M=\mathbb{R} \cup S^{1}$, where $S^{1} \subset \mathbb{R}^{2}$ is the unit circle. Let $N=\mathbb{R}$. Define $E: \operatorname{Diff}(M) \rightarrow \operatorname{Diff}(N)$ by $E(f)=f \mid \mathbb{R}$. Let $\pi: N \rightarrow M$ be the inclusion. Then $(M, N, E, \pi, \infty, 1)$ is a regular quasi-natural bundle such that $\pi$ is not surjective.

In connection with the implication $(4) \Rightarrow(1)$ of Proposition 1.1 we give the following two examples.

Example 2.4. Let $M=\mathbb{R} \times\{0\} \cup \mathbb{R} \times\{1\}$. We equip $N:=M$ with the topology generated by $N, \emptyset, \mathbb{R} \times\{0\}$ and $\mathbb{R} \times\{1\}$. Let $E: \operatorname{Diff}(M) \rightarrow$ Homeo $(N)$ be the inclusion and $\pi: N \rightarrow M$ the identity. It is clear that $(M, N, E, \pi, \infty, 1)$ is a quasi-natural bundle. If $f: M \rightarrow M$ is defined by $f(t, x)=(t, x+1(\bmod 2)), \varphi: M \rightarrow M$ is defined by $\varphi(t, x)=(-t, x)$, $v=(0,0)$ and $w=(0,1)$, then $w=f(v) \in \operatorname{Orb}(v)-\pi^{-1}(\pi(v)),\{v, w\}$ is Hausdorff in $N, \varphi \in D^{-}(\pi(v))$ and $\{E(\varphi)(v), w\}$ is also Hausdorff in $N$ but $\pi$ is not continuous at $v$, and $\pi(w) \notin \operatorname{Comp}(M, \pi(v))$.

Example 2.5. Let $M=\mathbb{R}$ and let $N$ be as in Example 2.4. Define $E: \operatorname{Diff}(M) \rightarrow \operatorname{Homeo}(N)$ by $E(f)(x, t)=(f(x), t)$ if $f$ is orientation preserving, and $=(f(x), t+1(\bmod 2))$ if $f$ is orientation reversing. Let $\pi: N \rightarrow M$ be the projection given by $\pi(x, t)=x$. It is easy to see that $(M, N, E, \pi, \infty, 1)$ is a quasi-natural bundle. If $v=(1,0)$ and $w=(-1,1)$, then $w=E(-\mathrm{id})(v) \in \operatorname{Orb}(v)-\pi^{-1}(\pi(v)), \pi(w) \in \operatorname{Comp}(M, \pi(v))$ and $\{v, w\}$ is Hausdorff in $N$ but $\pi$ is not continuous at $v$. Of course $\{E(\varphi)(v), w\}$ is not Hausdorff in $N$ for every $\varphi \in D^{-}(\pi(v))$.

With regard to the implication $(1) \Rightarrow(3)$ of Proposition 1.1 the following example is interesting.

Example 2.6. Let $M=\mathbb{R}$. We equip $N:=\mathbb{R} \times \mathbb{R}$ with the topology in which $U \subset N$ is open if and only if there exists an open subset $V$ of $\mathbb{R}$ such that $U=V \times \mathbb{R}$. Define $E: \operatorname{Diff}(M) \rightarrow \operatorname{Homeo}(N)$ by $E(f)(x, y)=$ $\left(f(x), \frac{d f}{d x}(x) y\right)$. Let $\pi: N \rightarrow M$ be the projection onto the first factor. Then $(M, N, E, \pi, \infty, 1)$ is a quasi-natural bundle. It is clear that $\pi$ is continuous at $v=(0,1)$ but $\operatorname{Orb}(v)$ is not Hausdorff in $N$.

In Example 2.7 we show that condition (3) of Proposition 1.1 cannot be replaced by the following one: If $u, w \in \operatorname{Orb}(v)$ and $\pi(u) \neq \pi(w)$, then $\{u, w\}$ is Hausdorff with respect to the induced topology.

ExAmple 2.7. Let $M=\mathbb{R}$. Endow $N:=\mathbb{R} \times \mathbb{R}$ with the minimal topology such that for all $\alpha, \beta \in \mathbb{R}, \mathbb{R} \times \mathbb{R}-((-\infty, \alpha] \times\{0\} \cup[\beta, \infty) \times\{0\})$ is open. Define $E: \operatorname{Diff}(M) \rightarrow \operatorname{Homeo}(N)$ by $E(f)(x, y)=(f(x), y)$. Let $\pi: N \rightarrow M$ be the projection onto the first factor. Then $(M, N, E, \pi, \infty, 1)$ 
is a quasi-natural bundle. Moreover, $\pi$ is not continuous at $v=(0,0)$ though for all $u, w \in \operatorname{Orb}(v)$ the condition $\pi(u) \neq \pi(w)$ implies that $\{u, w\}$ is Hausdorff with respect to the induced topology.

3. Regularity of projections. In this section we prove Theorem 0.3.

We shall use the following notation. For any smooth manifold $M$, we denote by $A(M)$ the Lie algebra of vector fields on $M$. We also consider $A(M)$ as a $C^{\infty}(M)$ module. For any $p \in M$ we write $M_{p}$ for $\left\{f \in C^{\infty}(M)\right.$ : $f(p)=0\}$. For any $p \in M$ we put $A_{p}=M_{p} A(M)$. Let $A^{0}=\{X \in A(M)$ : $\operatorname{supp}(X)$ is compact .

In the proof of Theorem 0.3 we use the following proposition.

Proposition 3.1. Let $(M, N, E, \pi, \infty, n)$ be a regular quasi-natural bundle. (Then $\operatorname{dim}(N)>0$.) Define $\widetilde{E}: A^{0}(M) \rightarrow A(N)$ by $\widetilde{E}(X)(y)=$ $\left[t \rightarrow E\left(\varphi_{t}\right)(y)\right]_{t=0}$, where $\left\{\varphi_{t}\right\}$ is the flow of $X$. Then $\widetilde{E}$ is a Lie algebra homomorphism.

Remark. For $\pi$ a $C^{\infty}$ submersion, this proposition is well known (see [12], [3]).

Proof of Proposition 3.1. If $X \in A^{0}(M), X(\pi(x)) \neq 0$ and $\left\{\varphi_{t}\right\}$ is the flow of $X$, then $\mathbb{R} \ni t \rightarrow E\left(\varphi_{t}\right)(x) \in N$ is a nontrivial curve, and thus $\operatorname{dim}(N)>0$. Therefore Proposition 3.1 is an immediate consequence of the following four lemmas.

LEMMA 3.1. The mapping $\widetilde{E}: A^{0}(M) \rightarrow A(N)$ is regular in the following sense: If $\widetilde{X}: U \times M \rightarrow T M$ is a $C^{\infty}$ mapping $\left(U\right.$ is a $C^{\infty}$ manifold) such that for all $\tau \in U$ the mapping $\widetilde{X}_{\tau}: M \rightarrow T M, \widetilde{X}_{\tau}(x)=\widetilde{X}(\tau, x)$, is an element of $A^{0}(M)$, then

$$
U \times N \ni(\tau, y) \rightarrow \widetilde{E}\left(\tilde{X}_{\tau}\right)(y) \in T N
$$

is also of class $C^{\infty}$.

Lemma 3.2. $\widetilde{E}: A^{0}(M) \rightarrow A(N)$ is $\mathbb{R}$-linear.

Lemma 3.3. For any $X \in A^{0}(M)$ and any $\varphi \in \operatorname{Diff}(M)$ we have $\widetilde{E}\left(\varphi_{*} X\right)$ $=(E(\varphi))_{*} \widetilde{E}(X)$.

LemMA 3.4. If $X, Y \in A^{0}(M)$, then $[\widetilde{E}(X), \widetilde{E}(Y)]=\widetilde{E}([X, Y])$.

Proof of Lemma 3.1. It is clear that $\widetilde{E}$ is local. Therefore since smoothness is also a local property, we may assume that there exists a compact set $K \subset U \times M$ such that $\widetilde{X}(\tau, x)=0$ for all $(\tau, x) \in U \times M-K$. Then we can consider $\widetilde{X}$ as an element of $A^{0}(U \times M)$. Let $\left\{\Phi_{t}\right\}$ be the global flow of $\tilde{X}$. Clearly, $\left\{\Phi_{t}\right\}$ is of the form $\Phi_{t}(\tau, x)=(\tau, \varphi(t, \tau, x))$, where $\varphi: \mathbb{R} \times U \times M \rightarrow M$ is of class $C^{\infty}$. It is easy to verify that for every $\tau \in U$, 
$\{\varphi(t, \tau, \cdot)\}$ is the flow of $\widetilde{X}_{\tau}$. From the regularity of $(M, N, E, \pi, \infty, n)$ it follows that

$$
U \times N \ni(\tau, y) \rightarrow[t \rightarrow E(\varphi(t, \tau, \cdot))(y)]_{t=0} \in T N
$$

is of class $C^{\infty}$.

Proof of Lemma 3.2. We fix $y \in N$ and $X, Y \in A^{0}(M)$. Define $E_{y}: \mathbb{R}^{2} \rightarrow T_{y} N$ by $E_{y}(\alpha, \beta)=\widetilde{E}(\alpha X+\beta Y)(y)$. If $\left\{\varphi_{t}^{\alpha, \beta}\right\}$ is the flow of $\alpha X+\beta Y$, then $\left\{\varphi_{\tau t}^{\alpha, \beta}\right\}$ is the flow of $\tau(\alpha X+\beta Y)$, and thus since $\left\{E\left(\varphi_{\tau t}^{\alpha, \beta}\right)\right\}$ is the flow of $\widetilde{E}(\tau(\alpha X+\beta Y))$ and $\left\{E\left(\varphi_{t}^{\alpha, \beta}\right)\right\}$ is the flow of $\widetilde{E}(\alpha X+\beta Y)$, we find that $\tau \widetilde{E}(\alpha X+\beta Y)=\widetilde{E}(\tau(\alpha X+\beta Y))$, where $\alpha, \beta, \tau \in \mathbb{R}$. Therefore for every $\alpha, \beta, t \in \mathbb{R}$ we have $E_{y}(t(\alpha, \beta))=t E_{y}(\alpha, \beta)$. It follows from Lemma 3.1 that $E_{y}$ is of class $C^{\infty}$. Hence $E_{y}$ is $\mathbb{R}$-linear.

Proof of Lemma 3.3. Let $X \in A^{0}(M)$ and $\varphi \in \operatorname{Diff}(M)$. If $\left\{\varphi_{t}\right\}$ is the flow of $X$, then $\left\{\varphi \circ \varphi_{t} \circ \varphi^{-1}\right\}$ is the flow of $\varphi_{*} X$, and so $\{E(\varphi) \circ$ $\left.E\left(\varphi_{t}\right) \circ(E(\varphi))^{-1}\right\}$ is the flow of both $\widetilde{E}\left(\varphi_{*} X\right)$ and $(E(\varphi))_{*} \widetilde{E}(X)$. Thus $\widetilde{E}\left(\varphi_{*} X\right)=(E(\varphi))_{*} \widetilde{E}(X)$.

Proof of Lemma 3.4. Let $X, Y \in A^{0}(M)$ and let $\left\{\varphi_{t}\right\}$ be the flow of $X$. If $F: \mathbb{R} \times M \rightarrow T M$ is given by $F(t, x)=\left(\left(\varphi_{t}\right)_{*} X\right)(x)-X(x)$, then (since $F(0, \cdot)=0$ ) there exists $\widetilde{F}: \mathbb{R} \times M \rightarrow T M$ of class $C^{\infty}$ such that $F(t, x)=t \widetilde{F}(t, x)$ for every $(t, x) \in \mathbb{R} \times M$. Therefore $\widetilde{X}: \mathbb{R} \times M \rightarrow T M$ given by

$$
\widetilde{X}(t, x)= \begin{cases}-\frac{\left(\left(\varphi_{t}\right)_{*} X\right)(x)-X(x)}{t}, & t \neq 0, \\ -\lim _{t \rightarrow 0} \frac{\left(\left(\varphi_{t}\right)_{*} X\right)(x)-X(x)}{t}, & t=0,\end{cases}
$$

is of class $C^{\infty}$. It follows from Lemmas 3.1-3.3 that

$$
\begin{aligned}
\widetilde{E}([X, Y])(y) & =\widetilde{E}\left(\lim _{t \rightarrow 0} \widetilde{X}_{t}\right)(y)=\lim _{t \rightarrow 0} \widetilde{E}\left(\widetilde{X}_{t}\right)(y) \\
& =\lim _{t \rightarrow 0} \widetilde{E}\left(-\frac{\left(\varphi_{t}\right)_{*} X-X}{t}\right)(y) \\
& =-\lim _{t \rightarrow 0} \frac{\left(\left(E\left(\varphi_{t}\right)\right)_{*} \widetilde{E}(X)\right)(y)-\widetilde{E}(X)(y)}{t} \\
& =[\widetilde{E}(X), \widetilde{E}(Y)](y) .
\end{aligned}
$$

Proof of Theorem 0.3. Let $\widetilde{E}: A^{0}(M) \rightarrow A(N)$ be the Lie algebra homomorphism defined in Proposition 3.1. Define a Lie algebra homomor$\operatorname{phism} \varphi: A(M) \rightarrow A(N)$ by $\varphi(X)(y):=\widetilde{E}(\widetilde{X})(y)$, where $X \in A(M), y \in N$ and $\widetilde{X} \in A^{0}(M)$ is such that $\operatorname{germ}_{\pi(y)}(\widetilde{X})=\operatorname{germ}_{\pi(y)}(X)$.

First we show that $N^{+}:=\left\{q \in N: \varphi^{-1}\left(A_{q}(N)\right) \neq A(M)\right\}=N$. Let $q \in N$ and let $X \in A^{0}(M)$ be such that $X(\pi(q)) \neq 0$. Then $\varphi(X)(q) \neq 0$, 
for if $\left\{\varphi_{t}\right\}$ is the flow of $X$, then there exists $\varepsilon>0$ such that $\varphi_{t}(\pi(q)) \neq \pi(q)$ for all $t \in(0, \varepsilon)$; hence $E\left(\varphi_{t}\right)(q) \neq q$ for all $t \in(0, \varepsilon)$, and thus $\varphi(X)(q) \neq 0$. We have proved that $N^{+}=N$.

By the results of K. Masuda [7, pp. 509-511] for every $q \in N^{+}$there exists a unique non-empty finite subset $\psi(q)=\left\{p_{1}, \ldots, p_{l}\right\}$ of $M$ such that $d / n \geq l$ and

$$
\bigcap_{i=1}^{l} M_{p_{i}} A(M) \supset \varphi^{-1}\left(A_{q}(N)\right) \supset \bigcap_{i=1}^{l} M_{p_{i}}^{h+1} A(M),
$$

where $h=2\left((d-n l)^{2}+d-n l\right)+1, d=\operatorname{dim} N$ and $n=\operatorname{dim} M$. We prove that $\psi(q)=\{\pi(q)\}$ for every $q \in N$.

Let $q \in N=N^{+}$. It is sufficient to show that $p \notin \psi(q)$ for every $p \in$ $M-\{\pi(q)\}$. Assume the contrary. Let $p \in M-\{\pi(q)\}$ with $p \in \psi(q)$. Then (in particular) $\varphi^{-1}\left(A_{q}(N)\right) \subset M_{p} A(M)$. On the other hand, if $X \in A^{0}(M)$ is such that $X(p) \neq 0$ and $\operatorname{germ}_{\pi(q)}(X)=\operatorname{germ}_{\pi(q)}(0)$, then $\varphi(X)(q)=0$ (i.e. $\left.X \in \varphi^{-1}\left(A_{q}(N)\right)\right)$ and $X \notin M_{p} A(M)$, a contradiction.

Therefore $\operatorname{int}\left\{q \in N^{+}: \operatorname{card} \psi(q)=1\right\}=N$. Hence by the results of K. Masuda [7, pp. 509-511], for every $q \in N$ there exist an open neighbourhood $U$ of $q$, an open dense subset $V \subset U$, an open neighbourhood $U_{1}$ of $\pi(q)$ and a continuous mapping $\widetilde{\psi}: U \rightarrow U_{1}$ such that $\widetilde{\psi} \mid V$ is a $C^{\infty}$ submersion and for any $q \in U$ the equality $\widetilde{\psi}(q)=p$ implies $\psi(q)=\{p\}$.

Problem. Let $(M, N, E, \pi, \infty, n)$ be a regular quasi-natural bundle. Is $\pi$ of class $C^{\infty}$ ?

4. Prolongation functors with compact fibres. In this section we prove Theorem 0.4. In the proof we use the following proposition which is similar to Proposition 14 of [13].

Proposition 4.1. (I) Let $F$ be a quasi-prolongation functor over $C^{r}$ manifolds such that conditions (1)-(3) of Theorem $0.4(\mathrm{I})$ are satisfied. Suppose that $f: P \times M \rightarrow N$ is of class $C^{r}$, where $M, N, P$ are $C^{r}$ manifolds. Then

$$
P \times F M \ni(t, x) \rightarrow F(f(t, \cdot))(x) \in F N
$$

is continuous.

(II) Let $F$ be a regular quasi-prolongation functor over $C^{\infty}$ manifolds such that condition (1) of Theorem $0.4(\mathrm{II})$ is satisfied. Suppose that $f$ : $P \times M \rightarrow N$ is of class $C^{\infty}$, where $M, N, P$ are $C^{\infty}$ manifolds. Then

$$
P \times F M \ni(t, x) \rightarrow F(f(t, \cdot))(x) \in F N
$$

is of class $C^{\infty}$. 
Proof of Proposition 4.1. Let $\left(t_{0}, x_{0}\right) \in P \times M$. Let $(U, g)$ be a chart of $P$ at $t_{0}$ and let $(V, h)$ be a chart of $M$ at $x_{0}$ such that $g(U)=\mathbb{R}^{p}$ and $h(V)=\mathbb{R}^{m}$, where $p=\operatorname{dim}(P)$ and $m=\operatorname{dim}(M)$. It is clear that $f(t, \cdot) \circ h^{-1}(y)=f \circ\left(g^{-1} \times h^{-1}\right) \circ \tau_{(g(t), 0)} \circ i(y)$ for every $(t, y) \in U \times \mathbb{R}^{m}$, where $i: \mathbb{R}^{m} \rightarrow \mathbb{R}^{p} \times \mathbb{R}^{m}$ is given by $i(y)=(0, y)$ and $\tau_{(g(t), 0)}: \mathbb{R}^{p} \times \mathbb{R}^{m} \rightarrow \mathbb{R}^{p} \times \mathbb{R}^{m}$ is the translation by $(g(t), 0)$. Therefore

$$
F(f(t, \cdot))(y)=F\left(f \circ\left(g^{-1} \times h^{-1}\right)\right) \circ F\left(\tau_{(g(t), 0)}\right) \circ F(i) \circ\left(F\left(h^{-1}\right)\right)^{-1}(y)
$$

for every $(t, y) \in U \times \pi_{M}^{-1}(V)$. On the other hand, for every $C^{r}$ manifold $\widetilde{N},\left(\widetilde{N}, F \widetilde{N}, F \mid \operatorname{Diff}(\widetilde{N}), \pi_{\widetilde{N}}, r, \operatorname{dim}(\widetilde{N})\right)$ is a quasi-natural bundle and $F \widetilde{N}$ is a Hausdorff space, and thus (by Theorem 0.1) $\pi_{\widetilde{N}}: F \widetilde{N} \rightarrow \widetilde{N}$ is continuous. Hence for every $n$ the restriction of $F$ to the category of $n$-dimensional $C^{r}$ manifolds and embeddings is a locally determined associated space such that $F \mathbb{R}^{n}$ is a second countable Hausdorff space. Therefore for every $n$ the mapping

$$
\mathbb{R}^{n} \times F \mathbb{R}^{n} \ni(x, y) \rightarrow F\left(\tau_{x}\right)(y) \in F \mathbb{R}^{n},
$$

where $\tau_{x}: \mathbb{R}^{n} \rightarrow \mathbb{R}^{n}$ is the translation by $x$, is continuous (see Proposition 1.1 of [8]). It follows that $U \times \pi_{M}^{-1}(V) \ni(t, y) \rightarrow F(f(t, \cdot))(y) \in F N$ is continuous. Since continuity is a local property, Proposition 4.1(I) is proved. The proof of Proposition 4.1(II) is similar. (We need the well-known theorem of Montgomery and Zippin [9] on continuous Lie group actions.)

The main difficulty in proving Theorem 0.4 is to show the following propositions.

Proposition 4.2. Suppose the assumptions of Theorem $0.4(\mathrm{I})$ are satisfied. Then $F$ is of order 0, i.e. if $f, g: M \rightarrow N$ are $C^{r}$ mappings of $C^{r}$ manifolds such that $f(x)=g(x)$ for some $x \in M$, then $F(f) \mid \pi_{M}^{-1}(x)=$ $F(g) \mid \pi_{M}^{-1}(x)$.

Proposition 4.3. Let $F$ be a regular quasi-prolongation functor over $C^{\infty}$ manifolds. Assume that $F$ is of order 0 and $F \mathbb{R}^{n}$ has a countable basis for every $n$. Then for every $C^{\infty}$ manifold $M, \pi_{M}: F M \rightarrow M$ is a $C^{\infty}$ submersion.

Remark. If $F$ is a regular quasi-prolongation functor over $C^{\infty}$ manifolds such that $F \mathbb{R}^{n}$ has a countable basis for every $n$, then from Proposition $4.1(\mathrm{II})$ and Theorem 0.3 it follows that if $F \mathbb{R}^{n}$ is connected, then for every $C^{\infty}$ manifold $M, \pi_{M}: F M \rightarrow M$ is of class $C^{\infty}$ on some open dense subset of $F M$. The answer to the question "Is $\pi_{M}$ of class $C^{\infty}$ ?" is unknown. Proposition 4.3 ensures that for $F$ of order 0 the answer is affirmative.

Proof of Proposition 4.2. Let $(U, h)$ be a chart of $M$ at $x$ such that $h(U)=\mathbb{R}^{m}$ and $h(x)=0$. It is sufficient to show that $F\left(f \circ h^{-1}\right)(z)=$ $F\left(g \circ h^{-1}\right)(z)$ for every $z \in \pi^{-1}(0)$, where $\pi=\pi_{\mathbb{R}^{m}}$. 
Let $z \in \pi^{-1}(0)$ and let $\varphi: \mathbb{R} \times \mathbb{R}^{m} \rightarrow \mathbb{R}^{m}$ be given by $\varphi(t, x)=t x$. Since $\varphi(t, \cdot): \mathbb{R}^{m} \rightarrow \mathbb{R}^{m}$ is a diffeomorphism for every $t \in \mathbb{R}-\{0\}$, we have $F(\varphi(t, \cdot))\left(\pi^{-1}(0)\right)=\pi^{-1}(0)$ for every $t \in \mathbb{R}-\{0\}$. In particular, there exists a sequence $y_{n} \in \pi^{-1}(0)$ such that $F(\varphi(1 / n, \cdot))\left(y_{n}\right)=z$ for every $n$. Since $\pi^{-1}(0)$ is compact and second countable, it is a metrizable compact space, and thus there exist $y \in \pi^{-1}(0)$ and a subsequence $n(k)(k=1,2, \ldots)$ of $(1,2, \ldots)$ such that $y_{n(k)} \rightarrow y$ as $k \rightarrow \infty$. Then $F(\varphi(1 / n(k), \cdot))\left(y_{n(k)}\right) \rightarrow$ $F(0)(y)$ as $k \rightarrow \infty$ because of Proposition 4.1(I). Since $\pi^{-1}(0)$ is Hausdorff, $F(0)(y)=z$. Therefore $F\left(f \circ h^{-1}\right)(z)=F\left(f \circ h^{-1}\right) \circ F(0)(y)=$ $F\left(f \circ h^{-1} \circ 0\right)(y)=F\left(g \circ h^{-1} \circ 0\right)(y)=F\left(g \circ h^{-1}\right)(z)$.

Proof of Proposition 4.3. First we prove that $\pi_{M}: F M \rightarrow M$ is of class $C^{\infty}$. It suffices to show that $f \circ \pi_{M} \in C^{\infty}(F M)$ for every $f \in C^{\infty}(M)$.

Let $Y \in A^{0}(M)$. Since $F$ satisfies the assumptions of Proposition 4.1(II), $\left(M, F M, F \mid \operatorname{Diff}(M), \pi_{M}, \infty, \operatorname{dim}(M)\right)$ is a regular quasi-natural bundle. Let $\widetilde{F}: A^{0}(M) \rightarrow A(F M)$ be the Lie algebra homomorphism described in Proposition 3.1 (with $F \mid \operatorname{Diff}(M)$ playing the role of $E$ ). In the proof of Theorem 0.3 we have shown that $\widetilde{F}(Y)(y) \neq 0$ for all $y \in \pi_{M}^{-1}(\{x \in M$ : $Y(x) \neq 0\})$. On the other hand, if $X \in A^{0}(M)$ is such that $X\left(x_{0}\right)=0$, then $\widetilde{F}(X)(z)=0$ for all $z \in \pi_{M}^{-1}\left(x_{0}\right)$, for if $\left\{\varphi_{t}\right\}$ is the flow of $X$ and $z \in \pi_{M}^{-1}\left(x_{0}\right)$, then $\varphi_{t}\left(x_{0}\right)=x_{0}$ for all $t \in \mathbb{R}$, and thus (since $F$ is of order 0 ) $F\left(\varphi_{t}\right)(z)=F\left(\operatorname{id}_{M}\right)(z)=z$ for all $t \in \mathbb{R}$, hence $\widetilde{F}(X)(z)=0$. Moreover, for every $y \in F M,\left(f-f\left(\pi_{M}(y)\right)\right) Y \mid \pi_{M}(y)=0$. Owing to these facts, we have $0=\widetilde{F}\left(\left(f-f\left(\pi_{M}(y)\right)\right) Y\right)(y)=\widetilde{F}(f Y)(y)-f\left(\pi_{M}(y)\right) \widetilde{F}(Y)(y)$ for every $y \in F M$, and hence $f \circ \pi_{M} \mid \pi_{M}^{-1}(\{x \in M: Y(x) \neq 0\})$ is of class $C^{\infty}$. Therefore $f \circ \pi_{M}$ is of class $C^{\infty}$.

To show that $\pi_{M}$ is a submersion, let $y \in F M$ and let $(U, g)$ be a chart of $M$ at $\pi_{M}(y)$ such that $g(U)=\mathbb{R}^{m}$ and $g\left(\pi_{M}(y)\right)=0$. Since $\pi_{M} \mid \pi_{M}^{-1}(U)=g^{-1} \circ \pi \circ\left(F\left(g^{-1}\right)\right)^{-1}$ where $\pi=\pi_{\mathbb{R}^{m}}$, it suffices to show that $\operatorname{rank}\left(d_{v} \pi\right)=m$ for every $v \in \pi^{-1}(0)$. Indeed, it follows from Proposition 4.1(II) that the mapping $\sigma: \mathbb{R}^{m} \rightarrow F \mathbb{R}^{m}, \sigma(x)=F\left(\tau_{x}\right)(v)$, is of class $C^{\infty}$. We see that $\pi \circ \sigma=$ id and $\sigma(0)=v$. Therefore $\operatorname{rank}\left(d_{v} \pi\right)$ $=m$.

We are now in a position to prove Theorem 0.4 .

Proof of Theorem 0.4. Let $G:=\pi_{\mathbb{R}}^{-1}(0)$. Then $G$ is a compact second countable space. If $F$ is regular it follows from Proposition 4.3 that $G$ is a $C^{\infty}$ manifold. For every $C^{r}$ manifold $M$ we define a mapping $I_{M}$ : $M \times G \rightarrow F M$ by $I_{M}(m, f)=F(m)(f)$, where $m: \mathbb{R} \rightarrow M$ is given by $m(x)=m$. We are going to prove that $\left\{I_{M}\right\}$ gives an isomorphism between ()$\times G$ and $F$. 
By Proposition 4.2, $F$ is of order 0. Therefore the mapping $T_{M}: F M \rightarrow$ $M \times G$ given by $T_{M}(v)=\left(\pi_{M}(v), F(0)(v)\right)$ is equal to $I_{M}^{-1}$. From Proposition 4.1 we know that $I_{M}$ is continuous (and of class $C^{\infty}$ if $F$ is regular). Similarly, it follows from Theorem 0.1 and Proposition 4.3 that $T_{M}$ is continuous (and of class $C^{\infty}$ if $F$ is regular). Hence $I_{M}$ is a homeomorphism (and a $C^{\infty}$ diffeomorphism if $F$ is regular).

We see that $p_{M}(m, f)=m=\pi_{M} \circ F(m)(f)=\pi_{M} \circ I_{M}(m, f)$ and $I_{N} \circ(() \times G)(g)(m, f)=I_{N}(g(m), f)=F(g(m))(f)=F(g \circ m)(f)=$ $F(g) \circ F(m)(f)=F(g) \circ I_{M}(m, f)$ for every $(m, f) \in M \times G$.

\section{References}

[1] J. Aczél und S. Gołąb, Funktionalgleichungen der Theorie der geometrischen Objekte, PWN, Warszawa 1960.

[2] D. B. A. Epstein and W. P. Thurston, Transformation groups and natural bundles, Proc. London Math. Soc. (3) 38 (1979), 219-236.

[3] J. Gancarzewicz, Differential Geometry, Bibl. Mat. 64, Warszawa 1987 (in Polish).

[4] - Liftings of functions and vector fields to natural bundles, Dissertationes Math. 212 (1983).

[5] M. Golubitsky and V. Guillemin, Stable Mappings and Their Singularities, Springer, New York 1973.

[6] I. Kolář, Functorial prolongations of Lie groups and their actions, Časopis Pěst. Mat. 108 (1983), 289-293.

[7] K. Masuda, Homomorphism of the Lie algebras of vector fields, J. Math. Soc. Japan 28 (1976), 506-528.

[8] W. M. Mikulski, Locally determined associated spaces, J. London Math. Soc. (2) 32 (1985), 357-364.

[9] D. Montgomery and L. Zippin, Transformation Groups, Interscience, New York 1955 .

[10] A. Nijenhuis, Natural bundles and their general properties, in: Diff. Geom. in honor of K. Yano, Kinokuniya, Tokyo 1972, 317-334.

[11] R. S. Palais and C. L. Terng, Natural bundles have finite order, Topology 16 (1978), 271-277.

[12] S. E. Salvioli, On the theory of geometric objects, J. Differential Geom. 7(1972), 257-278.

[13] J. Slovák, Smooth structures on fibre jet spaces, Czechoslovak Math. J. 36 (111) (1986), 358-375.

INSTITUTE OF MATHEMATICS

JAGIELLONIAN UNIVERSITY

REYMONTA 4

30-059 KRAKÓW, POLAND 